\title{
Self-tuning PID-type Fuzzy Adaptive Control for CRAC in Datacenters
}

\author{
Junwen Deng ${ }^{1}$, Liu Yang ${ }^{1}$, Xinrong Cheng ${ }^{2}$, and Wu Liu ${ }^{1}$ \\ ${ }^{1}$ College of Engineering, China Agricultural University, Beijing 100083, China \\ ${ }^{2}$ College of Information and Electrical Engineering, \\ China Agricultural University, Beijing 100083, China \\ $\{563106956,1151781485\} @$ @qq.com, yangliuacau.edu.cn
}

\begin{abstract}
In order to eliminate the current negative condition of Automatic Computer Room Air-Conditioning (CRAC) system, self-tuning Fuzzy Logic Control (FLC) was designed and applied to fan speed in CRAC system. In this paper, we derive a thermodynamic model of a datacenter suitable for applying adaptive self-tuning PID-type fuzzy adaptive control theory. It combines the classic PID control strategy and fuzzy adaptive control theory. The classic PID control uses the error and rate of change of error as its inputs to control the temperature automatically, and the fuzzy logic controller is used in the selftuning PID-type fuzzy control to tune the parameters of PID controller on-line by fuzzy control rules. Simulation and testing results show that the proposed self-tuning FLC method can achieve less steady-state error and short settling time in temperature control of datacenter.
\end{abstract}

Keywords: Air-Conditioning (CRAC) system, self-tuning Fuzzy control, adaptive control.

\section{$1 \quad$ Introduction}

Significant temperature pressure has been placed on traditional datacenters thermal management by increasing server power dissipation. Automatic Computer Room AirConditioning (CRAC) system improvement challenges have been steadily increasing over the past few years due to an increase in energy consumption and heat generation in datacenters. CRAC fan speeds have a significant effect on the recirculation flows in the datacenter, which are verified to be a major cause of inefficiency [1-3]. The energy needed for adjusting fan speed is much lower than the energy needed for adjusting the duty cycle of air compressor. For this reason, flexible design of CRAC fan speed optimization system supplying demand is substantially to reduce the energy consumption.

So far, classic control techniques, such as Proportional-Integral- Derivation (PID) controllers are still widely used in practice as they can be easily implemented, are low cost and reliable in harsh field condition. $K_{p}, K_{i}$ and $K_{d}$ parameters of PID were obtained to minimize the system error in many studies. 
Recently, some complex control strategies based on classical control concepts have been proposed in attempts to improve the system performances. Salsbury described a feed forward control scheme based on a simplified physical model as a supplement of the conventional PI feedback control [4]. Kasahara et al. proposed a robust PID control scheme to deal with the model uncertainty caused by the changing characteristics of the plant [5]. Bi and Cai et al. developed an advanced auto-tuning PID controller for both temperature and pressure control [6].

Classic control schemes commonly use the first order or second order plus time delay models to represent process dynamics. The performances of these control schemes are limited when applied to CRAC process due to their inherent nonlinearity and time varying nature. To overcome these drawbacks, applications of intelligent control to CRAC system have drawn some interests. Some authors proposed fuzzy PID methods to improve performance of the control system. In Shayeghi $H$, Shayanfar HA, and Jalili's pervious papers [7] and [8], the authors proposed an improved control strategy based on fuzzy theory and genetic algorithm (GA) technique have been proposed. Fuzzy logic (FLC) of heating, ventilating and airconditioning system was studied by Shan AJ, Krishnan N and Schmidt RR [7, 9]. The obtained results were comparing with those of PID control and these studies indicated that FLC had better result. However, the steady-state error was not totally eliminated. Existing work on thermal management in data centers, however, not take into account unexpected thermal anomalies and neglect the cost of control actions taken by the air conditioning system. This can lead to overheating of some spots. Obtaining better performances of the simulated systems became easier [10]. To eliminate this negative condition, self-tuning FLC was designed and applied to fan speed in CRAC system.

\section{Related Work}

In this paper, we derive a thermodynamic model of a data center suitable for applying adaptive self-tuning PID-type fuzzy adaptive control theory. We propose a novel control strategy based on this model. It combines the classic PID control strategy and fuzzy adaptive control theory. The classic PID control uses the error and rate of change of error as its inputs to control the temperature automatically and the fuzzy logic controller is used in the self-tuning PID-type fuzzy controller to tune the parameters of PID controller on-line by fuzzy control rules. It was shown that the usage of modeling and simulation methods for analyzing, testing and developing of CRAC systems decreases the design cost as well as the duration of the design process. Simulation and testing results show that the proposed self-tuning FLC method can achieve good performance in temperature control of datacenter. 


\section{The Thermal Model of the Datacenter}

Fig.1 shows the layout of datacenter and location of a blade. Datacenter design plays an important role in the efficient thermal management. Chilled water CRAC units supply a raised floor plenum underneath the racks with cold air. Perforated tiles are located near the racks to transfer the cool supply air to the front of the racks.

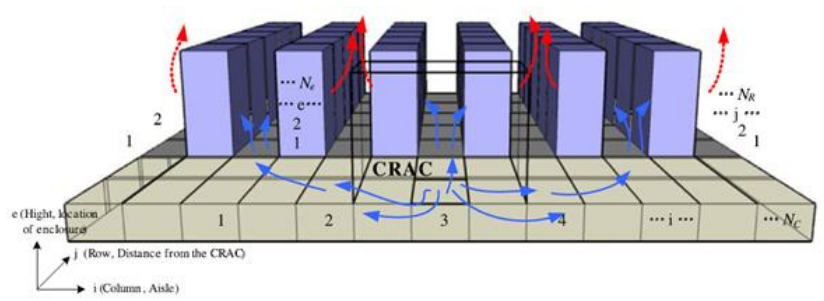

Fig. 1. The Layout of datacenter and location of a blade

As the CRAC system has featured such as considerable interference, non-linearity, large time delay, uncertainty and non-predicative, it is difficult to obtain the accurate mathematical model of thermodynamic in datacenter by considering all parameters. For this reason, we consider the following assumptions: According to the law of the energy conservation, the mathematical equation for datacenter thermal is as follows:

$$
\begin{gathered}
c_{a} v_{a} \rho_{a} \frac{d t_{d}}{d_{t}}=c_{a} \rho_{a} f_{a}\left(t_{d}-t_{a}\right)-A_{s} \mu_{s}\left(t_{s}-t_{d}\right)+q_{n}+q_{m} \\
q_{m}=c_{m} m_{m} \frac{d t_{m}}{d t}=\mu_{m} A_{m}\left(t_{m}-t_{d}\right)
\end{gathered}
$$

As most equipment in datacenter are made of irons, they have large coefficient of heat conductive, so we assume temperature variety rate has not much difference between equipment temperature variety rate.

$$
\frac{d_{m}}{d_{t}}=n_{m} \frac{d t_{d}}{d_{t}}
$$

If the fan motor heat dissipation is ignored, the equation (1) can be transferred to transfer function form as follows:

$$
\begin{aligned}
& c_{a} v_{a} \rho_{a} s-c_{m} m_{m} n_{m} s-c_{a} \rho_{a} f_{a} t_{d}-A_{s} d \\
& =-c_{a} \rho_{a} f_{a} t_{a}-A_{s} t_{s}
\end{aligned}
$$

If $c$ is defined as CRAC hybrid equivalent thermal capacity as follows:

$$
c=c_{m} m_{m} n_{m}-c_{a} v_{a} \rho_{a}
$$


And $R$ is defined as CRAC entire thermal resistance and $R_{s}$ is defined as Surrounding walls, floor and roof thermal resistance as follows:

$$
R_{S}=\frac{1}{A_{S} \mu_{S}}
$$

After standard arrangement the result is as follows:

$$
t_{d}(s)=\frac{k_{d} t_{a}(s)}{T s+1}+\frac{K_{s} t_{s}(s)}{T s+1}
$$

The time constant and other parameters are as follows:

$$
\begin{aligned}
T & =C^{*} R \\
K_{d} & =K^{*} R \\
K_{s} & =\frac{R}{R_{s}}
\end{aligned}
$$

As the air heat transfer delay affects the datacenter temperature, consider delay time is, the equivalent transfer to be as follows:

$$
t_{d}(s)=\frac{k_{d} t_{a}(s)}{T s+1} e^{-\tau s}+\frac{K_{s} t_{s}(s)}{T s+1} e^{-\tau s}
$$

Setting the real-time temperature in the datacenter is 27 , and the expected temperature in the datacenter is 20.The air flux of the datacenter was determined by the fan speed at end, Fan speed control is the core technology in Variable Air Volume (VAV) air condition system. It decides the liability and energy saving level of the CRAC system in the database. The fan speed is controlled by the open volume of the air condition. The transfer function of open volume to temperature can be expressed as an object of second-order inertial and net delay:

$$
G(S)=\frac{10 e^{-12 s}}{(1+20 s)(1+30 s)}
$$

The database CRAR system Mathematic Model is showed in figure 2. In the model, $U(t)$ is the output of the controller and $y(t)$ is the actual temperature of the database room.

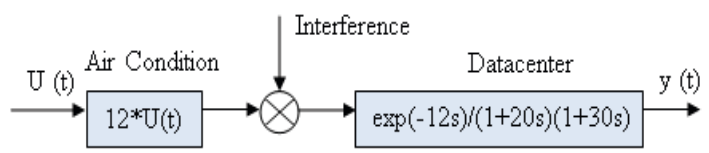

Fig. 2. CRAR System Mathematic Model 
Table 1. Notation

\begin{tabular}{|c|c|}
\hline & \\
\hline$C_{a}$ & Air specific heat $\left[\mathrm{J} / \mathrm{kg}^{\circ} \mathrm{C}\right]$ \\
\hline$\overline{V_{a}}$ & Air volume $\left[\mathrm{m}^{3}\right]$ \\
\hline $\bar{\rho}$ & Air density $\left[\mathrm{kg} / \mathrm{m}^{3}\right]$ \\
\hline$t_{d}$ & Real-time air temperature in dry test-bed [ $[\mathrm{C}]$ \\
\hline$f_{a}$ & Fan air volume delivery $\left[\mathrm{m}^{3} / \mathrm{s}\right]$ \\
\hline$t_{a}$ & AGDR air temperature $\left[{ }^{\circ} \mathrm{C}\right]$ \\
\hline$t_{s}$ & Temperature of surroundings [ $\mathrm{C}]$ \\
\hline$\overline{t_{m}}$ & Temperature of equipment [ $[\mathrm{C}]$ \\
\hline$A_{s}$ & Area of surroundings $\left[\mathrm{m}^{2}\right]$ \\
\hline$\overline{\mu_{s}}$ & Coefficient of convective heat exchange between surrounding and air $\left[\mathrm{w} /\left(\mathrm{m}^{2} \mathrm{C}\right)\right]$ \\
\hline$q_{n}$ & Fan motor heat dissipation $[\mathrm{w}]$ \\
\hline$q_{m}$ & Equipment heat dissipation [w] \\
\hline$c_{m}$ & Equipment specific heat $\left[\mathrm{J} /\left(\mathrm{kg}^{\circ} \mathrm{C}\right)\right]$ \\
\hline $\bar{m}$ & Equipment mass $[\mathrm{kg}]$ \\
\hline$\mu_{m}$ & Coefficient of convective heat exchange between equipment and air $\left[\mathrm{w} /\left(\mathrm{m}^{2} \mathrm{C}\right)\right]+$ \\
\hline$A_{m}$ & Area of equipment $\left[\mathrm{m}^{2}\right]$ \\
\hline$n_{m}$ & Coefficient of dry test-bed temperature variety rate to equipment temperature $[\mathrm{w} / \mathrm{C}]$ \\
\hline $\bar{C}$ & AGDR hybrid equivalent thermal capacity $[\mathrm{F}]$ \\
\hline$\underline{R}$ & AGDG entire thermal resistance $[\Omega]$ \\
\hline$R_{s}$ & Surroundings thermal resistance $[\Omega]$ \\
\hline
\end{tabular}

\section{Self-tuning PID-type Adaptive Control}

The control system is optimized through the whole working range of the process and ensures a maximum of control loop quality by a very short response time of any alternation in the process and at a negligible overshooting of the process value during the control phase. The fuzzy controller showed enormous advantages in processed with intensive nonlinearity. An incremental fuzzy logic controller is used in the selftuning PID-type fuzzy controller to tune the parameters of PID controller on-line by fuzzy control rules. The controller uses the error and rate of change of error as its inputs and can meet the desire of self-tuning parameters based on time-varying $e$ and $\dot{e}$, and the output of the fuzzy controller is $\mathrm{u}$.

$$
u(k)=K_{p} e(k)+k_{i} \sum^{k} e(i)+k_{d}[e(k)-e(k-1)]
$$


The database CRAR system Mathematic Model is showed in Fig.2. In the model, $U(t)$ is the output of the controller and $\mathrm{y}(\mathrm{t})$ is the actual temperature of the database room. Where $k_{p}$ is the controller gain.

$$
K_{d}=\frac{k_{p} T}{T_{i}}
$$

$T$ is the sample time; $T i$ is the integral time parameter, $T_{d}$ is the derivative time parameter [11]. The sub-block of language was given in the following manner: Positive Big (PB), Positive Middle (PM), Positive Small (PS), Zero (ZO), Negative Small (NS), Negative Middle (NM), Negative Big (NB). The output of the fuzzy systems is $\mathrm{u}$. The fuzzy variables are defined for the rule base as: (e, $\dot{e})=\{$ the error, the variation of error $\},\{\mathrm{NB}, \mathrm{NS}, \mathrm{Z}, \mathrm{PS}, \mathrm{PB}\} . k_{p}, k_{d}, k_{i}=\{$ the control parameters, Z, PS,PM, PB, PVB (Positive Very Big), [0,1], 1\}. Fig.3 shows the rule base fuzzy PID-type control and the rule $K i$ fuzzy.
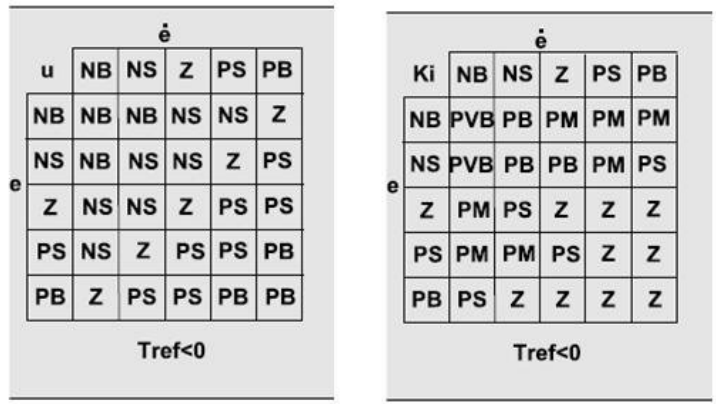

Fig. 3. (a) Shows the rule base fuzzy (b)Shows the rule $K i$ fuzzy

A membership function is a curve that defines how each point in the input space is mapped to a membership value (on degree of membership) between 0 and 1 . In our study we will employ the trapezoidal membership functions for each fuzzy linguistic value of error e and the change rate of error $\dot{e}$ showed in Fig.4.

By applying the self-tuning PID-type fuzzy adaptive controller, the control of the same CRAC system has been realized in order to compare of the control performance. The classical PID and FLC theories are combined in this study. The $K_{p}, K_{i}, K_{d}$ values of PID parameters have adaptively been determined by using the dynamic FLC for each time-step. In this case, FLC has two inputs $(e, \dot{e})$ and three outputs $\left(K_{p}, K_{i}, K_{d}\right)$. The physical domain of the inputs $(e, \dot{e})$ is $-1,-0.8,-0.6,-0.4,-0.2,0,0.2,0.4,0.6$, $0.8,1$ and that of the outputs $\left(K_{p}, K_{i}, K_{d}\right)$ is $0,0.1,0.2,0.3,0.4,0.5,0.6,0.7,0.8,0.9,1$, selected again based on trial and error. Fig.5 shows the membership functions of output $\mathrm{u}$. 


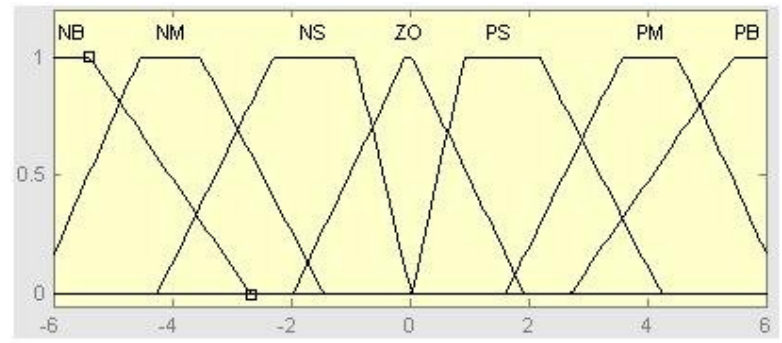

Fig. 4. The membership functions for the input e and $\dot{e}$

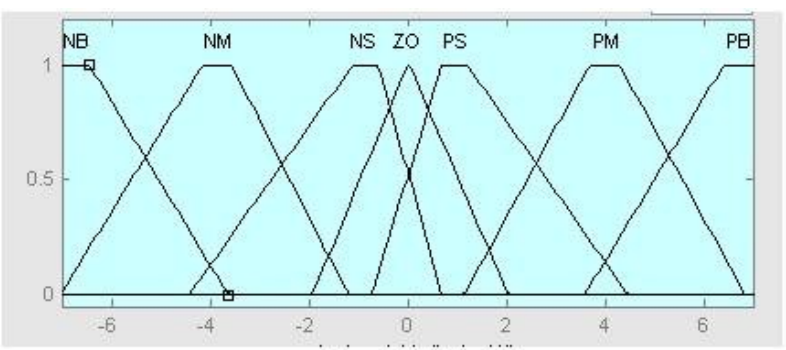

Fig. 5. The membership functions for the output $u$

\section{The Numerical Simulation of the CRAC System}

By using MATLAB/SIMULINK package program, the control, model and numerical simulation of CRAC fan system have been realized. The control diagram of CRAC fan system has been obtained by considering the input and output values of the device and the desired datacenter conditions. The obtained results have been presented in a graphical form. Every PID control system has its parameter range. It will result in unreliability and out of control if the parameters are out of the range. In the CRAC control system, the PID parameters are tuned by fuzzy inference. According to simulation, the tuning range of parameter $K_{p}$ is between $[25.4,40.5]$, the tuning range of parameter $K_{i}$ is between [0,0.5] and the tuning grange of parameter $K_{d}$ is between $[15.2,20.6]$.If the system is overshoot, first decrease the parameter $K_{p}$, then decrease the parameter $K_{i}$ and in the end decrease the parameter $K_{d}$. If the system has long settling time, first decrease the parameter $K_{i}$, then decrease the parameter $K_{p}$ and in the end decrease the parameter $K_{d}$. If the system has short settling time and High frequency fluctuations, first decrease the parameter $K_{d}$, then decrease the parameter $K_{p}$ and in the end decrease the parameter $K i$. The considered CRAC system controlled by the fuzzyPID type and self-tuning PID-type fuzzy adaptive controller is shown in the block diagram Fig.6. $T_{r e f}$ is the desired reference temperature and $\mathrm{T}$ is the real temperature in datacenter. The PID parameters are tuned by adaptive fuzzy controller. Fig.6 shows the diagram of self-tuning fuzzy-PID control simulation system of CRAC fan. PID control model, fuzzy PID model [12] and self-tuning fuzzy-PID control model are respectively 
established in the control link of the room temperature control and are contrasted through the MATLAB simulation [13]. Transferred discrete PID controller module from the sub-library of Simulink [14], after repeated experiments, took the ratio coefficient $K_{p}=171$, integral time constant $K_{i}=0$. 001, differential time constant $K_{d}=10$ in PID control system [15]. The design idea of fuzzy-PID control is to find out the fuzzy relationship between 3 parameters of fuzzy- PID control and deviation $e$, change rate of deviation $\Delta e$, continuous detection $e$ and calculation the $\Delta e$ during the operation and online modified control parameters in accordance to the control principle, to meet the different requirements in different $e$ and $\Delta e$, so that the controlled object has a good dynamic and static properties. We choose the set point to react based on the temperature in which ranges from $64.4 \mathrm{~F}\left(18^{\circ} \mathrm{C}\right)$ to $80.6 \mathrm{~F}\left(27^{\circ} \mathrm{C}\right)$.

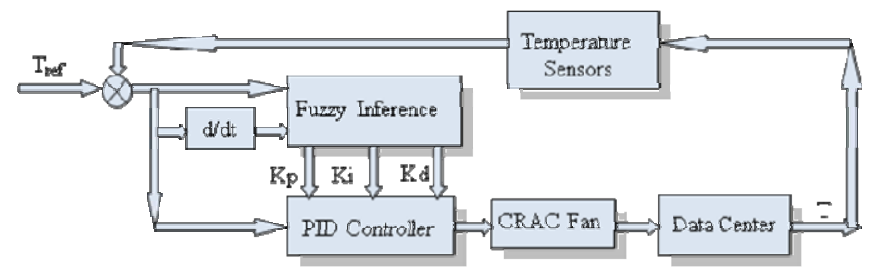

Fig. 6. The control diagram of CRAC fan system

Fig.7 shows the temperature control of datacenter when the classical PID, fuzzyPD type and the self-tuning PID-type fuzzy adaptive controller are applied [16]. To simulate and compare facilitate the control model, set the supposed temperature for 25.5 , simulate time for the $1500 \mathrm{~s}$.

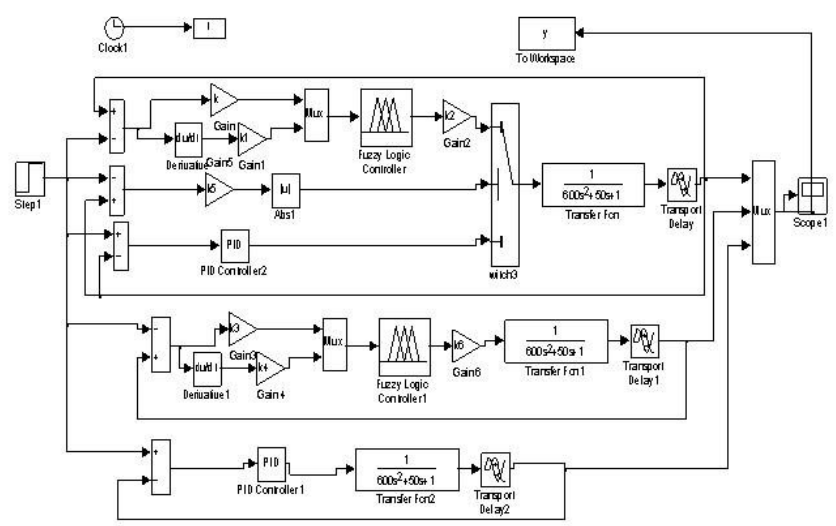

Fig. 7. The diagram of self-tuning fuzzy-PID control, fuzzy-PID control and PID control simulation

Fig.8 shows the simulation results: (1) PID control. More sensitive to interference [17], control is not satisfactory, with a large overshoot. The simulation found that there will be instability if the interference is too large. (2) General fuzzy-PID control. 
Subjective the control rules, there is no self-learning function. The simulations found that the domain of fuzzy controller had been changed, required different parameters and bigger deviations in different given temperatures. (3) Self tuning Fuzzy-PID control. Self-tuning Fuzzy-PID control can achieve better control [18], shorter response time, small overshoot, and with good response performance and robustness, and can achieve better control.

The performance of the self-tuning PID-type fuzzy adaptive controller is the best among the others in terms of both the steady-state error and the settling time. There is no steady-state error [19] and the system reaches the desired reference temperature [20] while minimizing settling time. Although the fuzzy-PD type controller has no steady-state error, it has longer settling time. The classical PID controller has the worst performances.

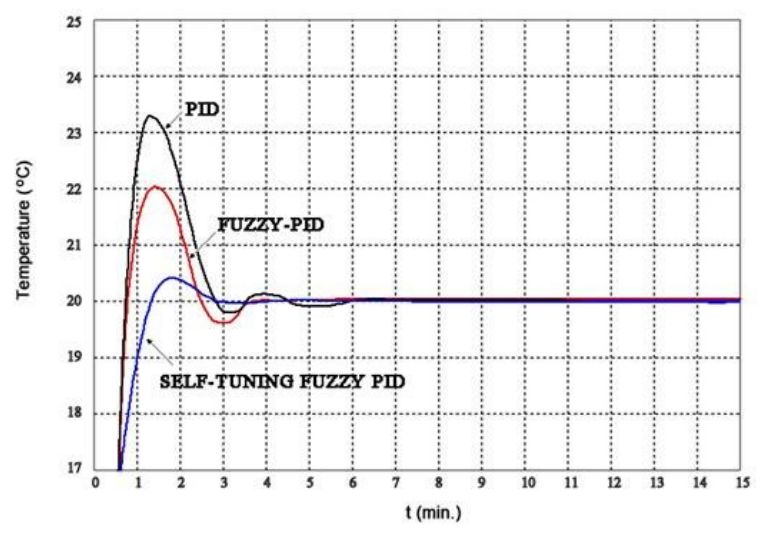

Fig. 8. The temperature control of datacenter

Fig.9 shows $K_{p}-K_{i}-K_{d}$ variation of datacenter when the self-tuning PID-type fuzzy adaptive controller is applied.
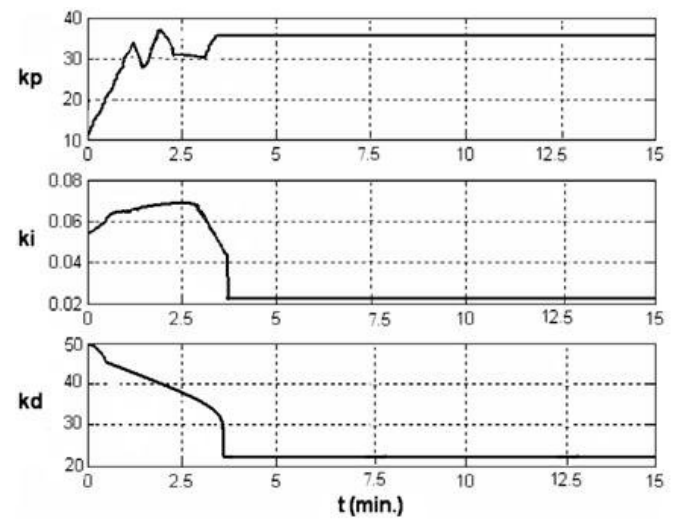

Fig. 9. Tuning trajectories of PID controller gains 


\section{Conclusion}

Over the course of this study three types of CRAC fan speed controller were realized (and tested in the data center) by using MATLAB/SIMULINK pack-age program namely, the PID controller, the fuzzy-PD controller and self-tuning fuzzy PID controller. The result shows that the self-tuning fuzzy PID controller is the best among the others, in terms of both the steady-state error and the settling time.

\section{References}

1. Bash, C.E., Patel, C.D., Sharma, R.K.: Efficient Thermal Management of Data Centers Immediate and Long-Term Research Needs. Intl. J. HVAC\&R. Res. (2003)

2. Boucher, T.D., Auslander, D.M.: Viability of Dynamic Cooling Control in a Data Center Environment (2004)

3. Lee, E.K., Kulkarni, I., Pompili, D., Parashar, M.: Proactive Thermal Management in Green Datacenter. The Journal of Super-computing (2010)

4. Salsbury, T.I.: A temperature controller for VAV air-handing units based on simplified physical models. HVAC and R. Res. (1998)

5. Kasahara, M., Matsuba, T., Boucherand, T.D., Auslander, D.M.: Design and tuning of robust PID controller for HVAC system (1999)

6. Bi, Q., Cai, W.-J., Wang, Q.-G.: Advanced controller auto-tuning and its application in HVAC system (2000)

7. Shayeghi, H., Shayanfar, H.A., Jalili, A.: Multi stage fuzzy PID power system automatic generation controller in the deregulated environment. Energy Convers Manage (2006)

8. Shayeghi, H., Jalili, A., Shayanfar, H.A.: Robust modified GA based multi-stage fuzzy LFC. J Energy Convers Manage (2007)

9. Schmidt, R.R.: Thermal profile of a high-density data center-methodology to thermally characterize a data center. Trans Am Soc Heat RefrigAirCondEng, ASHRAE (2004)

10. Schmidt, R.R., Karki, K.C., Patankar, S.V.: Raised floor computer data center: perforated tile flow rates for various tile layouts Thermal profile of a high-density data centermethodology to thermally characterize a data center. In: Proc. of Intersociety Conference on Thermal Phenomena in Electronic Systems, ITHERM (2004)

11. Tao, Y.Y.H., New-style, G.Y.X.: PID control and application (2001)

12. Lei, L., Wang, H., Yu, Y.: Adaptive Fuzzy PID Control Method Based on Identification Structure. International Journal of System and Control (2006)

13. Yang, Y.: Application of MATLAB in PID Control Theory's teaching Reform. Journal of Changshu Institute of Technology (2009)

14. Wang, S., Jiang, W.: PID Tuning Based on MATLAB/Simulink. Industry Control and Applications (2009)

15. Lu, R.: Matter-element Modeling of Parallel Structure and Application about Extension PID Control Syetem. Joural of Systems Science \& Complexity (2006)

16. Wang, L., Du, W.: Fuzzy self-tuning PID control of the operation temperatures in a twostaged membrane separation process. Journal of Natrual Gas Chemistry (2008) 
17. Zi, B., Duan, B., Qiu, Y.: Fuzzy-PID control base on disturbance observer and its application.Systems Engineering and Electronics (2006)

18. Ma, Y., Liu, Y., Wang, C.: Design of Parameters Self-tuning Fuzzy PID Control for DC Motor. In: The 2nd International Conference on Industrial Mechatronics and Automation (ICIMA 2010) (2010)

19. Yu, Q.: Based on MATLAB's Analysis of Steady-State Error in Control System. Journal of Changshu College (2004)

20. Liu, Y.: Study on boiler temperature system PID controlbased on RBF neural network. Journal of Baoji University of ArtsandSciences (Natural Science) (2011) 